\title{
Book Review: Handbook of Learning Analytics
}

\author{
Paul R. Bowdre, Ed.D. \\ Oakland City University \\ 138 N. Lucretia Street \\ Oakland City, IN 47660 \\ USA
}

The Handbook of Learning Analytics, edited by Charles Lang, George Siemens, Alyssa Wise, and Dragan Gašević(2017), proposes to meet the different requirements of a growing and new field. The book focuses on balancing quality, rigor, breadth of appeal, and open access. The book was written to act as an introduction to and an overview of the recent state of research. The different sections and chapters found in the book were peer-reviewed by various members who are committed in these fields and also printed with the support and commendation of the International Society for Educational Data Mining and the Society for Learning Analytics Research. This book is an excellent resource because it incorporates different editors and writers who are prominent practitioners and researchers of educational data mining and learning analytics to deliberate on some of the most important questions which are regularly not adequately understood by people who do not qualify as experts in the field of learning analytics.

This book was dedicated to and written in memoriam of Erik Duval. Duval's strong convictions and passion acted as the foundation on which the field and community of the learning analytics were created. Additionally, Duval's scientific contribution and participation in the learning analytics community made him a leading expert. He was loved for his openness, integrity, personality, and constant curiosity. All the authors and editors of this book got their inspiration from Erik's ability to openly share research and ideas. The main aim of this handbook was to animate the discussion associated with the field of learning analytics and also increase access to its various sub-domains and areas. The authors and publishers of this book wanted to aid student learning and also help in the improvement of various education systems.

The handbook was meant to codify practices and ideas within the growing fields related to educational data mining and learning analytics. It also acts as a reference, which might help to promote the consistency of the work that is being carried out and expand its effect by encouraging the accessibility of different vital ideas. The publication has been successfully shaped with the help of the various core demands associated with the production of a comprehensive resource, which could assist in teaching different contents at the mastery level.

The handbook has four different sections that deal with various perspectives associated with learning analytics. The four sections include Foundational Concepts, Techniques and Approaches, Strategies, and Systems Perspectives and Institutional Strategies. The first section, which is the Foundational Concepts, serves as a starting point for those people who are not familiar with the domain and also examines different high-level concepts. The first chapter of the book, by Simon Buckingham Shum and Simon Knight, discusses the benefits associated with theory generation concerning learning analytics. Other vital topics that are analyzed in the first section include the application and history of computational methods and the provision of basic coverage on educational psychometrics and measurement associated with learning analytics. The last chapter in the first section considers the ethical implications related to learning analytics practice and research.

The second section, which is Techniques and Approaches, provides an insight into the development of appropriate methodologies within the field of learning analytics. The second section starts with reviewing predictive modeling with the help of Craig Thompson and Chris Brooks. Kenneth Koedinger and Ran Liu expand on the introduction to predictive modeling and highlights the differences between explanatory and predictive models. The second chapter of the second section by George Siemens, Vitomir Kovanović, Dragan Gašević, Marek Hatala, and Srećko Joksimović provides information on different data sources and also covers the developing content analytics sub-field. The theme related to unstructured data is examined with a review and introduction of Natural Language Processing by Laura Allen, Scott Crossley, Cecile A. Perret, Mihai Dascalu, and Danielle McNamara. Sydney D'Melloexplores the effect emotional learning analytics have within computational sciences and the learning. The topic of multimodal can cover the different expansive ways through which student data can be traced and used. The process involved in using and taking up analytic tools is also discussed. The third section, Applications, examines the various ways through which methodologies may be applied within the framework of learning analytics. This section also considers the perspectives of students using an introduction to ways through which data-driven feedback may impact their performance. 
This section gives a detailed introduction about the audiences, goals, and uses associated with analytic dashboards. Andrew Ruis and David Shaffer present the use of theory-based approaches through the use of an epistemic network analysis example. The description of a system called TRACES is also illustrated for models that are related to sociotechnical network data by Daniel Suthers. Applications associated with the Big Data realm are discussed in three chapters. Mark Rosenstein and Peter Foltz deliberate on large scale writing assessments. Christopher Brooks and René Kizilcecwere able to analyze randomized experiments. Predictive modeling with the help of granular action data was also carried out, and automated predictions were further established in a recommender systems tutorial by Hendrik Drachsler, SoudeFazeli, and Peter Sloep.Phil Winne was able to provide a detailed introduction to self-regulated learning through the utilization of learning analytics. The third section also considers the complexities associated with investigating the manipulation of user video. Lastly, the third section takes into account adult learners by examining professional learning analytics.

The handbook's final section on Systems Perspectives and Institutional Strategies will assist readers in understanding and appreciating various practical challenges associated with the implementation and application of learning analytics. Three chapters in this section discuss the different aspects relevant to the realization of learning mature analytics ecosystem. This section discusses the various processes associated with developing virtual learning infrastructures. The challenges related to the utilization of different learning analytics are also discussed. It also examines a critical stance about the legitimacy of learning analytics and educational data mining for claiming and measuring outcomes in learning and educational settings. A comprehensive analysis of the state of student privacy was provided by ElanaZeide. The final section mostly discusses linked data, covering the use of the LAK dataset and considering the benefits associated with the improvement of education.

The handbook is a good source of information on learning analytics because it goes beyond evaluating the different learning analytics concepts, selected applications, best techniques for the study of several types of educational data, and various success stories from people in the field. The book incorporates different editors and writers who are prominent practitioners and researchers of educational data mining and learning analytics to deliberate on some of the critical questions which are not adequately understood by people who do not qualify as experts. Some of the chapters in the book were able to invite readers to reflect and analyze the integrally multidisciplinary research associated with learning analytics and concerning the value of considering educational psychology. The publication recognized theories related to educational research and learning sciences instead of only emphasizing the data-driven path. Some of the chapters were able to highlight the benefits associated with understanding and appreciating the difference between predictive and explanatory modeling or prescriptive and predictive analytics. Such information is beneficial to people who are thinking of or responsible for linking learning analytics with different policy-making processes, deploying it in an institution, or coming up with various personalized learning ideas.

This book also introduces the field of learning analytics to newcomers and welcomes old friends. The information in the book helps different researchers to discover inquiries which will excite them with ideas concerning how they may change the concept of education while frustrating them with the evidence of some of the challenges that people may face in the process. One of the weaknesses of the book is that it does not provide a broad spectrum of accountability and ethical issues related to learning analytics. It is vital for readers of the book to evaluate the broad range of ethical problems and responsibilities associated with learning analytics. Generally, the book is an excellent reference for those who are looking for information concerning learning analytics and those individuals who are working within the field of education who are searching for useful content to improve the quality of education.

The book has helped in the growth of the body of research relevant to the learning analytics field. It serves as a recognized resource about learning analytics for instructors, students, administrators, industry leaders, and practitioners. This volume offers an introduction and a framework for shaping and organizing different ideas and concepts in terms of techniques and approaches, foundational concepts, systems perspectives and institutional strategies, and applications. This book also offers an extensive view of what people know from the leading experts' perspective son learning analytics. The authors of the book have helped to make the book a good read for many people because they have helped to enrich and broaden the field of learning analytics by introducing new methodologies, theories, and concepts. The different readers of this book will not only find those chapters with conceptual and technical topics, but also those that address various sensitive and significant issues that emerge from research while making use of educational data such as ethics of data, student privacy, and institutional challenges that result from initiatives of national and local learning analytics. This handbook provides significant educational support. The different authors who participated in writing the book have managed to share their ideas and thoughts while promoting the discussion of new topics. The book is supposed to assist newcomers in understanding the extent to which the field and concepts associated with learning analytics may and can support students and educators. 
The fact that some of the ideas presented in the book are important in identifying some of the ways learning analytics can potentially harm and mislead teachers and students; it qualifies as a good read. This book also helps in the growth of educational data science and learning analytics. People who have the chance to read this book will gain a more holistic and more in-depth understanding of what being accountable, ethics-aware, and transparent is all about and how they can achieve that. With more volumes, this handbook will pass as a librarian buying book for a collection. Personally, this is one of the best books on learning analytics that I have read because it incorporates different theories and concepts that can help to promote education and features some of the best authors who are knowledgeable in different areas. The book acts as an essential instrument that drives further development of learning analytics and unlocks its full potential.

In conclusion, this book is a good read because it incorporates different editors and writers who are prominent practitioners and researchers of educational data mining and learning analytics to deliberate on some of the critical questions which are regularly not adequately understood by people who do not qualify as experts in the field of learning analytics. With different publications on this similar topic, learning analytics' future will be brighter. This book has helped to highlight some of the promising opportunities that learning analytics can offer for advancing people's understanding of various learning processes in several educational settings. Some of the things that learning analytics can offer include advising students, providing interventions in remedial education that are analytics-driven, understanding the success factors of students, and promoting better degree planning and curriculum modeling. By having a read-through of the handbook, people can understand how institutions and companies can develop educational software, including educational games, intelligent tutors, and learning management systems. The book also provides an analysis of different tools that have some elements associated with bettering the education system. This handbook is important because it helps people to collect useful educational data and gain insight into the effectiveness of learning and educational processes. This volume offers an introduction and a framework for shaping and organizing different ideas and concepts. The information in the book explains that if people have the right tools, the process of utilizing learning analytics produces the best results. Generally, this book is significant because it helps to educate learning analytics about the findings and techniques from different sets of data and their limits. I would recommend this book to newcomers so that they can understand how different concepts can support learners and educators. People who have the chance to read this book will gain a more holistic and more in-depth understanding of what being accountable, ethicsaware, and transparent is all about.

\section{Reference}

Lang, C., Siemens, G., Wise, A., \&Gašević, D. (Eds.). (2017). Handbook of learning analytics. SOLAR, Society for Learning Analytics and Research. doi: 10.18608/hla17 